\title{
Terahertz imagers based on metamaterial structures monolithically integrated in standard CMOS technologies
}

Ivonne Escorcia, James P. Grant, Luis Gouveia, David R. S. Cumming

Ivonne Escorcia, James P. Grant, Luis Gouveia, David R. S. Cumming, "Terahertz imagers based on metamaterial structures monolithically integrated in standard CMOS technologies," Proc. SPIE 10656, Image Sensing Technologies: Materials, Devices, Systems, and Applications V, 1065617 (14 May 2018); doi: 10.1117/12.2304335

Event: SPIE Commercial + Scientific Sensing and Imaging, 2018, Orlando, Florida, United States 


\title{
Terahertz imagers based on metamaterial structures monolithically integrated in standard CMOS technologies
}

\author{
Ivonne Escorcia ${ }^{\mathrm{a}}$, James P. Grant ${ }^{\mathrm{a}}$, Luis Gouveia ${ }^{\mathrm{b}}$, David R.S. Cumming*a \\ ${ }^{a}$ Microsystem Technology Group, School of Engineering, University of Glasgow, Scotland, United

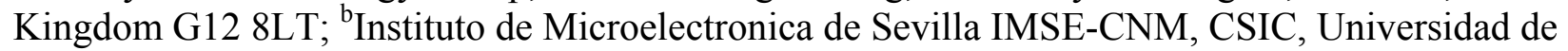 \\ Sevilla, Américo Vespucio, Sevilla, Spain 41092
}

\begin{abstract}
Silicon complementary metal oxide semiconductor (CMOS) technologies are arguably the most important asset in the world of electronics. Focal plane arrays (FPAs) are one of the driving forces in the revolution of low-cost, mass produced, compact, and high-resolution imaging devices. The importance of these imaging systems in the visible spectrum has highlighted the need of their implementation into other significant electromagnetic regions such as infrared (IR) and Terahertz (THz). The unique characteristics of $\mathrm{THz}$ waves make them ideal for a variety of important applications ranging from security and medical imaging, explosive and drug detection, and non-destructive quality control testing. These applications are possible due to the non-ionizing nature of $\mathrm{THz}$ radiation, its transparency to many non-conductive materials, and distinctive spectroscopic fingerprints of a vast number of substances.

Current $\mathrm{THz}$ imaging systems are usually restricted to laboratory use due the lack of compact, portable and roomtemperature operated sources and detectors. Therefore, the implementation of CMOS based THz detectors is key to promote the exploitation of low-cost, room-temperature, high-resolution, highly sensitive, and portable $\mathrm{THz}$ imaging systems. Here we present the monolithic integration of two types of THz FPAs fabricated in a standard $180 \mathrm{~nm} \mathrm{CMOS}$ process. The imagers are composed of $\mathrm{THz}$ metamaterials (MM) absorbers coupled to a microbolometer, either vanadium oxide (VOx) or silicon pn diode, integrated with readout electronics to form 64 x 64 CMOS FPAs. The suitability of $\mathrm{THz}$ imagers for stand-off imaging of concealed objects was demonstrated in transmission mode by capturing images of metallic objects hidden in a manila envelope.
\end{abstract}

Keywords: CMOS Terahertz detectors, Terahertz imaging, Terahertz metamaterials, CMOS image sensors, uncooled bolometers

\section{INTRODUCTION}

Image sensors based on solid-state electronics, such as charge-couple devices (CCDs) and metal oxide semiconductor (MOS) active-pixel sensors, pioneered the way for digital cameras. The small-scale integration of digital electronics based on silicon complementary metal oxide semiconductor (CMOS) technologies led to the creation of compact, lowcost, mass produced and high-resolution light-sensitive sensor arrays. The success of CMOS image sensors led to their inclusion into everyday life visible light applications. The most remarkable example is the evolution of the mobile phone cameras. From the initial resolution of $0.1 \mathrm{MP}$ it rapidly evolved to $12 \mathrm{MP}$ resolution and now $63 \mathrm{MP}$ resolution is commonplace for panoramic photos. All this became possible thanks to the efficient miniaturizing of the photodetectors using CMOS technology. Their implementation as mass-produced sensors with megapixel resolution raised the interest to integrate them to other wavelengths.

Thermal imaging in the IR spectrum rapidly benefited from the implementation of CMOS image sensors to detect minimal temperature differences, instead of light, in a scene. Initially they were only used by the military to obtain images in total darkness. Once uncooled microbolometers were integrated with CMOS electronics, the manufacturing price decrease and the volume production increased making them available for a wide variety of commercial and consumer applications. Such has been the rapid scaling and reduction in cost of IR cameras that FLIR, the world's leading manufacturer, now offer a 64 x 64 FPA that can be attached to a smart phone for only a few hundred dollars ${ }^{1}$.

The region of the electromagnetic spectrum found next to IR waves is known as Terahertz radiation. It lies in the frequency range between $100 \mathrm{GHz}$ to $10 \mathrm{THz}$ with respective wavelengths of $3 \mathrm{~mm}$ to $30 \mu \mathrm{m}$. This frequency range has

Image Sensing Technologies: Materials, Devices, Systems, and Applications V, edited by

Nibir K. Dhar, Achyut K. Dutta, Proc. of SPIE Vol. 10656, 1065617 · @ 2018 SPIE

CCC code: $0277-786 \mathrm{X} / 18 / \$ 18 \cdot$ doi: $10.1117 / 12.2304335$ 
also been known as ' $\mathrm{THz}$ gap' because it has been relatively unexplored compared to other regions of the electromagnetic spectrum. Originally, $\mathrm{THz}$ waves were only used for astronomy studies due to the lack of naturally existing sources on Earth. During the last 20 years, substantial effort has been invested to develop better sources and detectors due to the very promising characteristics of $\mathrm{THz}$ waves. So far, this spectrum has been used for many different applications such as security imaging, medical diagnosis and non-destructive quality testing. One of the main characteristics of $\mathrm{THz}$ is its transparency to many common materials such as paper, cardboard, plastic, ceramics and clothes. This important property allows for non-invasive package inspection and airport security screenings to detect potentially dangerous objects hidden inside any of the previously mentioned materials. The transparency of THz waves in conjunction with their extreme sensitivity to water content and non-ionizing radiation make $\mathrm{THz}$ ideal for medical imaging and biological studies to detect contrasts in healthy and unhealthy tissue without, in contrast to x-rays, being exposed to harmful radiation. In terms of imaging applications, $\mathrm{THz}$ waves provide better spatial resolution than millimeter waves due to their shorter wavelengths. In spectroscopy, THz technology has been used for the detection of illicit drugs and explosives due to their unique spectral $\mathrm{THz}$ fingerprints generally absent in the optical and X-ray regimes.

The two modalities of THz imaging, active and passive, have encountered challenges due to the lack of practical devices for generation and detection of $\mathrm{THz}$ waves. Passive detection of $\mathrm{THz}$ waves is restricted due to the low emissivity radiation of the objects and low sensitivity of room temperature operated detectors. Although cryogenically cooled and heterodyne detectors have overcome these limitations, they are expensive and bulky limiting them to laboratory use. Active detection suffers from the lack of cost-effective, portable, high-power sources and highly sensitivity, highresolution, fast, uncooled terahertz detectors. All these limitations have hindered the proliferation of $\mathrm{THz}$ applications into everyday life. In order to overcome these obstacles, research is focusing on creating imaging systems without the need for cryogenic cooling thus reducing component size and cost. One main solution is to create CMOS compatible $\mathrm{THz}$ detectors able to detect $\mathrm{THz}$ waves at room temperature thus reducing the fabrication cost and size. Additionally, CMOS integration also overcomes the challenge of scaling two dimensional array detectors (FPAs) required for highresolution imaging.

Technologies such as antenna-coupled bolometers and Field Effect Transistor (FET) detectors lead the way in highly sensitive CMOS THz detectors. Their high sensitivity allows them to detect $\mathrm{THz}$ waves with low power sources since they have a NEP in the range of nano to picowatts. CEA Leti have developed a 320 x 240 bolometric pixel array based on antenna-coupled bolometers operating at $2.5 \mathrm{THz}^{2}$. Their technology is based on bowtie antennas in quarterwavelength cavities, adopting the identical approach as is currently done for IR detectors. The company INO developed a THz camera with $384 \times 288$ uncooled microbolometer FPA operating at 4.25 to $0.094 \mathrm{THz}^{3}$. Their technology is based on bow-tie antennas fabricated as MM absorbers. Their work expands to the creation of $\mathrm{THz}$ cameras using metallic absorbers and antenna-coupled microbolometers. STMicroelectronics created a THz camera operating from 0.6 to $1 \mathrm{THz}$ made of FETs. These transistors surpass the cutoff frequency and absorb THz waves via a copper ring antenna connected to the transistor's source. The company NEC developed a bolometer based technology operating at frequencies from 1 to $7 \mathrm{THz}$ however sales of the unit were discontinued in 2016. Perhaps this is an indication of the need to find a unique and disruptive application for $\mathrm{THz}$ imaging that will increase the demand for $\mathrm{THz}$ cameras. A summary of a range of detector technologies that operate at room temperature can be found in "The 2017 Terahertz science and technology roadmap".

The work presented in this paper demonstrates the monolithic integration of two types of microbolometer sensors integrated with MM absorbers into a commercial $180 \mathrm{~nm}$ CMOS process to develop FPAs with front-end readout integrated circuits (ROICs). The integration of MM absorbers into the CMOS process was possible due to their layer structure which is composed of metal-insulator-metal. The CMOS integration of the FPA with minimal post-processing steps provides a low-cost $\mathrm{THz}$ detector able to function at room temperature with the great advantage of being easily incorporated into a high-resolution imaging system. An imaging application is demonstrated by capturing an image in transmission mode. The detector demonstrates the ability of $\mathrm{THz}$ radiation to penetrate non-conductive materials by imaging a metallic object hidden in a manila envelope.

The paper is organized as follows: an overview of the design of two types of monolithically integrated CMOS THz detectors is provided in Section 2. Detector characterization techniques to determine important figures of merit are described in Section 3. The results of the THz imaging experiments in transmission mode are shown in Section 4. Lastly, Section 5 describes an improved pixel design to increase the $\mathrm{THz}$ response of the detector and the work done to create a 64 x 64 FPA.

*David.Cumming.2@glasgow.ac.uk; phone+44 (0) 1413305858; www.gla.ac.uk/mst 


\section{DETECTOR DESIGN}

The monolithic integration of $\mathrm{THz}$ detectors into CMOS technologies is a key step to promote the exploitation of lowcost, room-temperature and portable $\mathrm{THz}$ imaging systems. The structure of $\mathrm{MM}$ absorbers, typically composed of metal-insulator-metal, lends itself to integration into the layers of a commercial CMOS process. Additionally, MMs were used due to their unique ability to absorb at any electromagnetic wavelength ranging from microwave to visible light. MMs gained popularity, especially in the THz spectrum, due to the lack of natural existing materials able to absorb $\mathrm{THz}$ radiation. MM absorbers are arrays of sub-wavelength elements meaning that they can absorb wavelengths larger than a single MM cell. They are composed of electric ring resonators (ERRs) or split ring resonators (SRRs) and ground plane fabricated in metallic layers separated by an insulating layer. Their electromagnetic properties are customized not by the material composition but by the size, shape and thickness of the insulating layer. One of the most important benefits of MMs structure is the design scalability allowing them to absorb at the frequency of choice $e^{5-7}$.

The proposed THz detector is composed of three major components: a broadband MM absorber, a microbolometer and readout electronics monolithically integrated in a standard six metal layer, 180nm, CMOS process from Texas Instruments. The broadband MM absorber is composed of three ERRs embedded in the metal-insulator layers of the CMOS process. The ERRs were integrated in metal 6 (M6), metal 5 (M5) and metal 4 (M4); each separated by the respective insulating layer, silicon dioxide $\left(\mathrm{SiO}_{2}\right)$, and a ground plane in metal 3 (M3). The geometric parameters of the ERRs are as follows: M4 cross arm length $=24.5 \mu \mathrm{m}$, M5 cross arm length $=23.5 \mu \mathrm{m}$, M6 cross arm length $=22.5 \mu \mathrm{m}$, and cross arm width $=2.5 \mu \mathrm{m}$.

a)

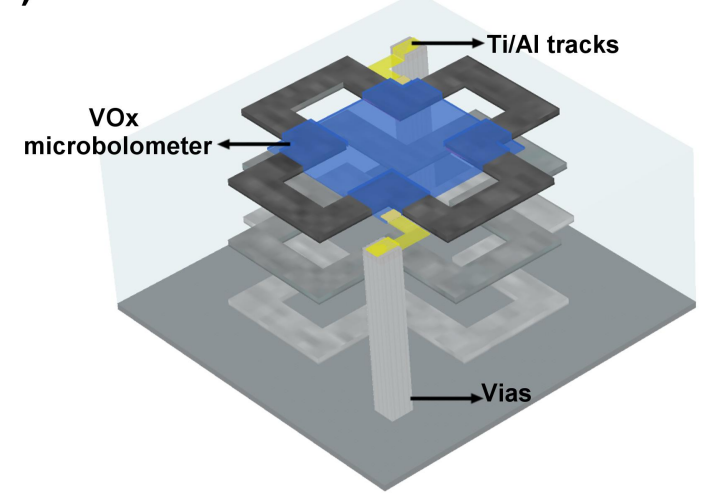

b)

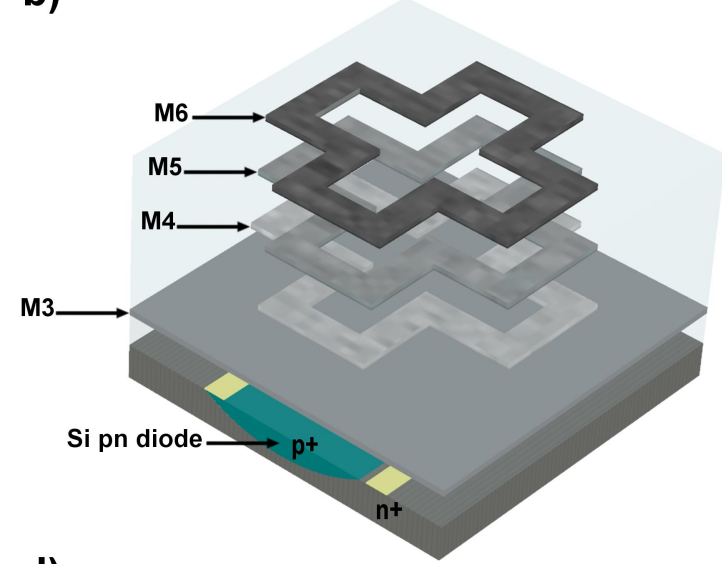

d)

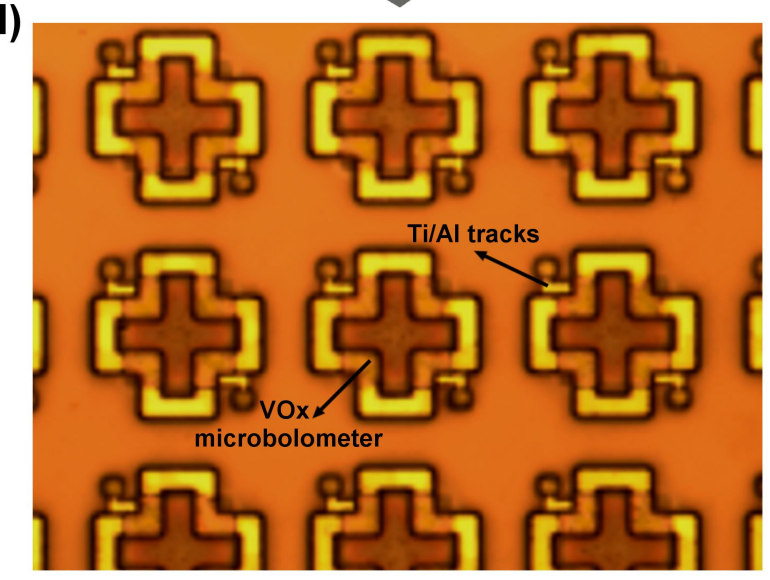

c)

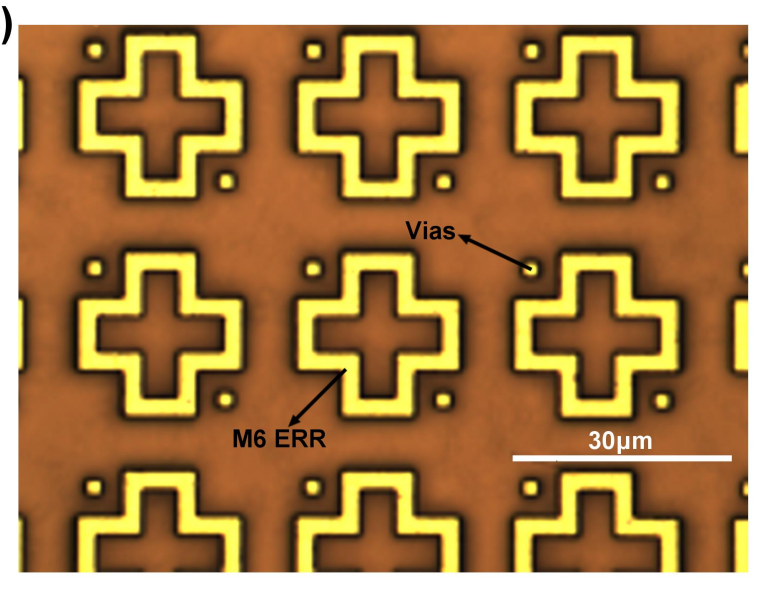

Figure 1. Schematics of broadband $\mathrm{THz}$ metamaterial absorbers composed of three layers of electric ring resonators embedded in a $180 \mathrm{~nm}$ CMOS process with the integration of a) a VOx microbolometer and b) Si pn diode. c) Optical image of a CMOS chip as received from the foundry and d) optical image of a completed post-processed chip with VOx microbolometers connected to the readout electronics using Ti/Al tracks and a stack of vias. 
Microbolometers were coupled with the MM absorbers to be able to quantify the THz absorption. Two uncooled microbolometer materials were investigated: VOx and silicon pn diode. Both options were investigated because of their sensitivity to temperature changes and wide use in IR imaging systems. The detection mechanism is based on sensing the heat generated by the incident $\mathrm{THz}$ wave and converting it to a current or voltage to be sensed by the readout electronics. The VOx (resistive film) has a temperature coefficient resistance (TCR) of $-2 \% / \mathrm{K}$. Minimal post-processing steps were required to pattern the VOx microbolometers above the M6 ERR structure. The VOx is connected to the readout electronics with Titanium/Aluminum (Ti/Al) tracks and vias from M6 to M2. A 3D schematic of the VOx detector is shown in Figure 1a. The pn diode has temperature coefficient of voltage (TCV) of $-2 \mathrm{mV} / \mathrm{K}$. It has the great advantage of being already integrated in the lower layers of the CMOS process, therefore no post-processing, apart from back etching of the substrate to reduce the thermal conductance and improve the responsivity, is needed. The pn diode detector 3D schematic is shown in Figure 1b. In order to increase the sensitivity of the pn diode detector, three diodes in series were integrated in every pixel to obtain an expected TCV of $-6 \mathrm{mV} / \mathrm{K}$. An optical image of the CMOS chip as received from the foundry is shown in Figure 1c. The post-processed chip with the VOx microbolometer and Ti/Al tracks connecting the readout electronics through the vias is shown in Figure 1d. The pixel's geometric parameters were: pixel size = $30 \mu \mathrm{m} \times 30 \mu \mathrm{m}$; bolometer thickness $=300 \mathrm{~nm}$; and Ti/Al tracks thickness $=1000 \mathrm{~nm}$.

The refractive index cross-section in the $x z$ plane and at $y=0$ of the materials composing the CMOS broadband $\mathrm{THz}$ absorber is shown in Figure 2a. The metallic layers from the three ERRs with an arm width of $2.5 \mu \mathrm{m}$ are shown in yellow as well as the ground plane. The dielectric insulating layers are shown in light blue and the air is shown in darker blue, hence a refractive index of 1 . The absorption characteristics of the broadband MM absorber were simulated using 3D finite difference time domain (FDTD) software ${ }^{8}$, Lumerical Inc. The spectral response of the THz broadband metamaterial absorber is shown in Figure 2b, the simulated response showing an $88.2 \%$ absorption at $2.65 \mathrm{THz}$ is shown in blue and the experimental response in red. The experimental results were obtained by placing the devices under vacuum in a Bruker IFS 66v/S Fourier Transform Infrared (FTIR) spectrometer. The experimental response shows two peaks: an absorption of $57 \%$ at the required frequency of $2.5 \mathrm{THz}$ (gray dashed line) and $70 \%$ at $2.81 \mathrm{THz}$.
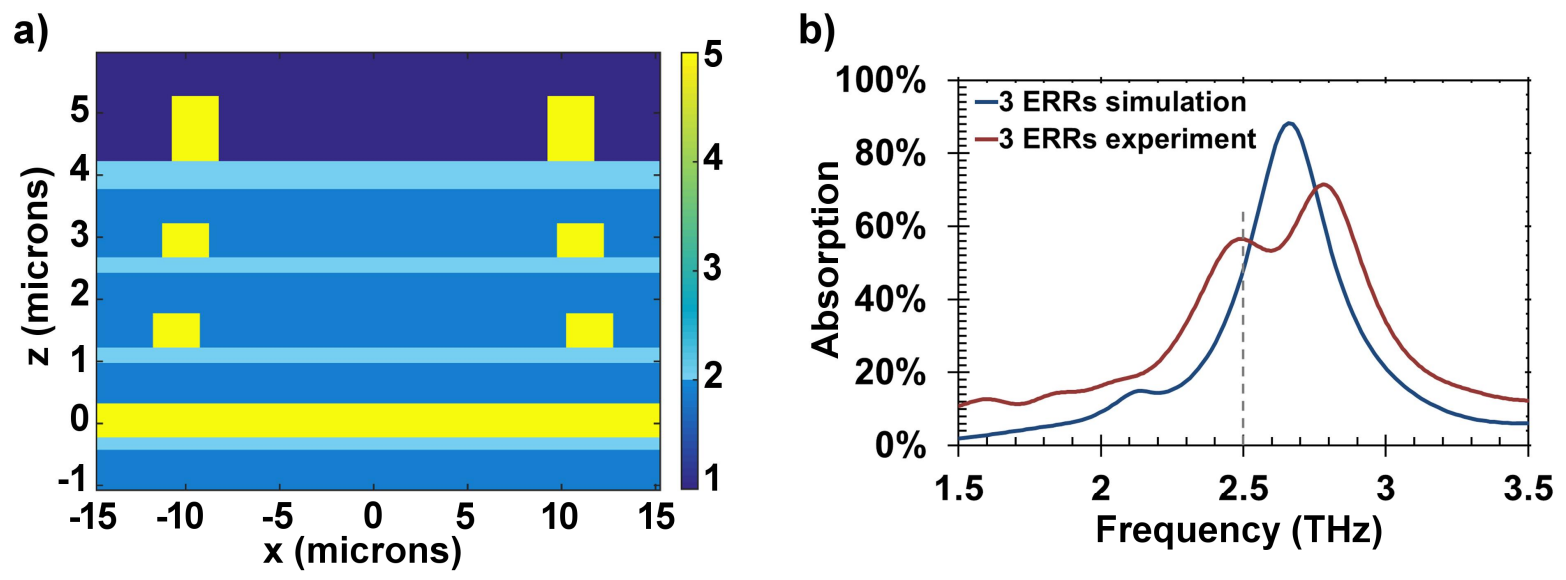

Figure 2. a) Refractive index cross section in the $x z$ plane and at $y=0$ of the broadband $\mathrm{THz} \mathrm{MM}$ absorber. The metallic layers of the ERRs and ground plane are shown in yellow, the insulating layers are shown in light blue and the air in darker blue. b) Spectral response of the $\mathrm{THz}$ broadband metamaterial absorber showing the simulated response (blue) and experimental response (red). The experimental response shows an absorption of $57 \%$ at $2.5 \mathrm{THz}$ (gray dashed line).

\section{DETECTOR'S FIGURES OF MERIT}

The detectors were characterized to obtain relevant figures of merit (FOM) including AC and DC responsivity, NEP, TCR and TCV and thermal time constant $(\tau)$. The active THz source used to characterize the detectors was a $\mathrm{CO}_{2}$ pumped methanol vapor laser operating at $118.8 \mu \mathrm{m}(2.5 \mathrm{THz})$, thus the need to create absorbers operating at this frequency. Figure 3 shows the set-up schematic used to determine the detector response and NEP. The DC responsivity of both detectors was characterized by biasing the VOx and pn diode at a range of bias currents using a Keithley 4200 SMU. The differential voltage was obtained by turning the laser on and off. The largest responsivity for the VOx detector with an $8 \mu \mathrm{m}$ membrane thickness was $32.65 \mathrm{kV} / \mathrm{W}$ obtained with a $600 \mathrm{nA}$ bias current. The largest responsivity of the pn diode detector with a $9 \mu \mathrm{m}$ membrane thickness was $684 \mathrm{~V} / \mathrm{W}$ for a $10 \mu \mathrm{A}$ bias current ${ }^{9}$. The TCR 
and TCV were obtained by placing the sensors in an environmental chamber and measuring at a range of temperatures and bias currents. The VOx sensor had an average room temperature $\left(25^{\circ} \mathrm{C}\right) \mathrm{TCR}$ of $-2 \% / \mathrm{K}$ and the Si pn diode had a $\mathrm{TCV}$ of $-5.4 \mathrm{mV} / \mathrm{K}$.

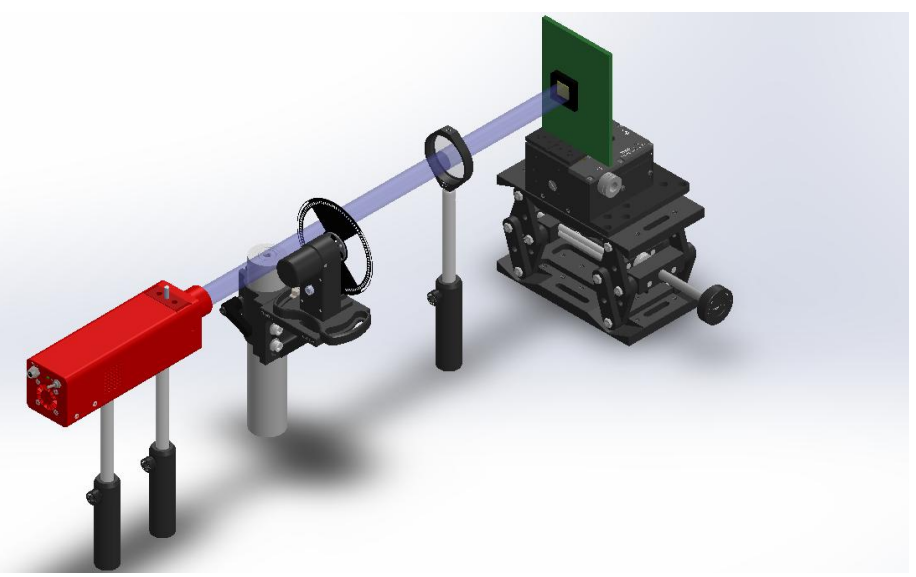

Figure 3. Schematic of set-up to determine the detector responsivity and noise equivalent power.

The AC responsivity was measured at different frequencies up to $50 \mathrm{~Hz}$ for the VOx sensor and up to $10 \mathrm{~Hz}$ for the pn diode. Figure $4 \mathrm{a}$ shows the responsivity versus modulation frequency demonstrating the largest responsivity at $1 \mathrm{~Hz}$ for both sensors. For the VOx sensor (red squares), the responsivity was $59 \mathrm{kV} / \mathrm{W}$ (bias current of $2 \mu \mathrm{A}$ ) and for the pn diode (blue diamonds) the responsivity was $274 \mathrm{~V} / \mathrm{W}$ (bias current of $10 \mu \mathrm{A}$ ). The thermal time constant was obtained from the responsivity versus frequency data and it was found to be $68 \mathrm{~ms}$ for the VOx sensor and $420 \mathrm{~ms}$ for the pn diode. The NEP was obtained by measuring the root-mean-square $(\mathrm{rms})$ noise output voltage per square root unit bandwidth of the sensor divided by the responsivity modulated at different frequencies. Figure $4 \mathrm{~b}$ shows the NEP from the VOx sensor (red squares) having a minimum NEP at $15 \mathrm{~Hz}$ of $108 \mathrm{pW} / \sqrt{\mathrm{Hz}}$ and a minimum NEP of $10.4 \mathrm{nW} / \sqrt{\mathrm{Hz}}$ at $5 \mathrm{~Hz}$ for the pn diode (blue diamonds). The performance of the VOx and Si pn diode detectors is compared in Table $1^{10}$.

a)

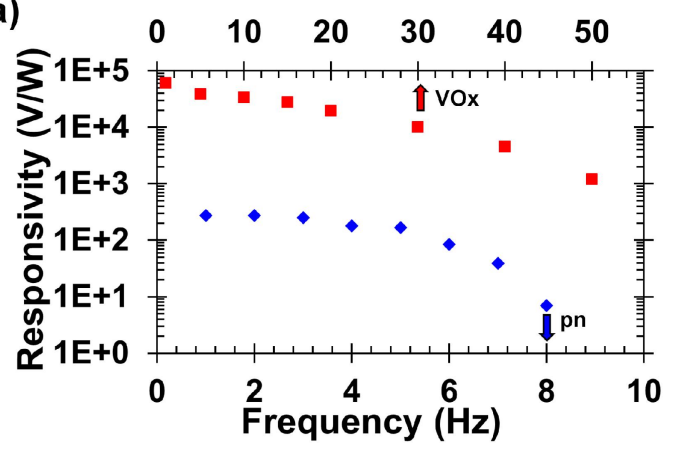

b)

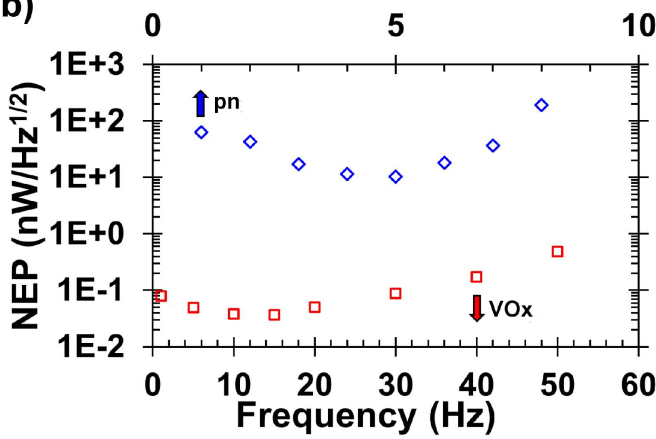

Figure 4. Comparison between VOx (red) and Si pn (blue) detectors a) responsivity as a function of frequency and b) NEP.

Table 1. Figures of merit comparison from both $\mathrm{THz}$ detectors.

\begin{tabular}{|c|c|c|}
\hline Figures of Merit & VOx $^{11}$ & Si pn $^{12}$ \\
\hline $\mathrm{TCR} / \mathrm{TCV}$ & $-2 \% / \mathrm{K}$ & $-5.4 \mathrm{mV} / \mathrm{K}$ \\
\hline $\mathrm{DC} \mathrm{R}_{v}$ & $32.6 \mathrm{kV} / \mathrm{W}(600 \mathrm{nA})$ & $683.7 \mathrm{~V} / \mathrm{W}(10 \mu \mathrm{A})$ \\
\hline $\mathrm{AC} \mathrm{R}_{v}$ & $59 \mathrm{kV} / \mathrm{W}(1 \mathrm{~Hz}-2 \mu \mathrm{A})$ & $274 \mathrm{~V} / \mathrm{W}(1 \mathrm{~Hz}-10 \mu \mathrm{A})$ \\
\hline Membrane thickness & $8 \mu \mathrm{m}$ & $9 \mu \mathrm{m}$ \\
\hline $\mathrm{NEP}$ & $108 \mathrm{pW} / \sqrt{\mathrm{Hz}}(15 \mathrm{~Hz})$ & $10.4 \mathrm{nW} / \sqrt{\mathrm{Hz}}(5 \mathrm{~Hz})$ \\
\hline$\tau$ & $68 \mathrm{~ms}$ & $420 \mathrm{~ms}$ \\
\hline
\end{tabular}




\section{IMAGING EXPERIMENTS}

The VOx detector was employed in a transmission mode single pixel imaging experiment using a CW FIR gas laser operating at $2.5 \mathrm{THz}$ as the active source. This detector was used due to having the best performance compared to the pn diode detector. An aluminum object in a "UoG" shape shown in Figure 5a was imaged. The object was attached to a holder and placed on a motorized $x-y-z$ translation stage. The object was located at the focal point of a TPX lens (focal length $=4 \mathrm{~cm}$ ) and an identical lens was place after the object to collimate the beam. A Tsurupica lens with a focal length of $11 \mathrm{~cm}$ was used to focus the transmitted beam into the VOx detector. The incident THz beam was modulated using an optical chopper set to $1 \mathrm{~Hz}$. The detector was biased at $100 \mathrm{nA}$ and the output voltage was read by a lock-in amplifier. A Labview program was used to acquire the differential data read by the lock-in amplifier measured at every $0.5 \mathrm{~cm}$ in the $x$ and $y$ direction. Since the object was larger than the range of the stages, the captured image shown in Figure $5 b$ is the result of merging three frames together (one for each letter). The object was hidden in a manila envelope (Figure 5c) and the resulting image is shown in Figure 5d. The image of the hidden object shows a better signal to noise ratio because the lock-in amplifier thermal time constant was increased from 1 second to 3 seconds. The variation from pixel to pixel is mainly due to the instability of the laser, nonetheless, the letters in both images are clearly discernible.

a)

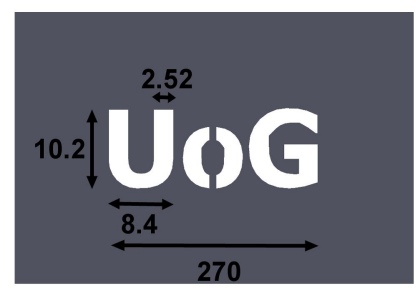

c)

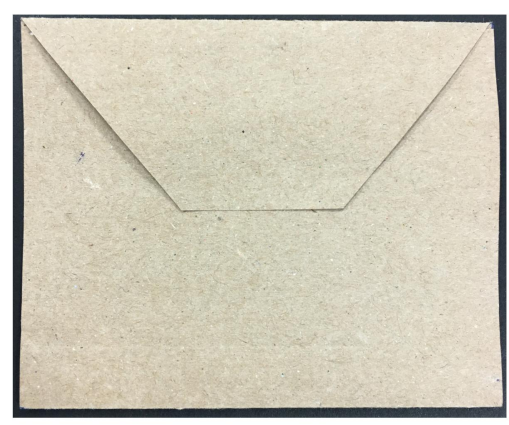

b)

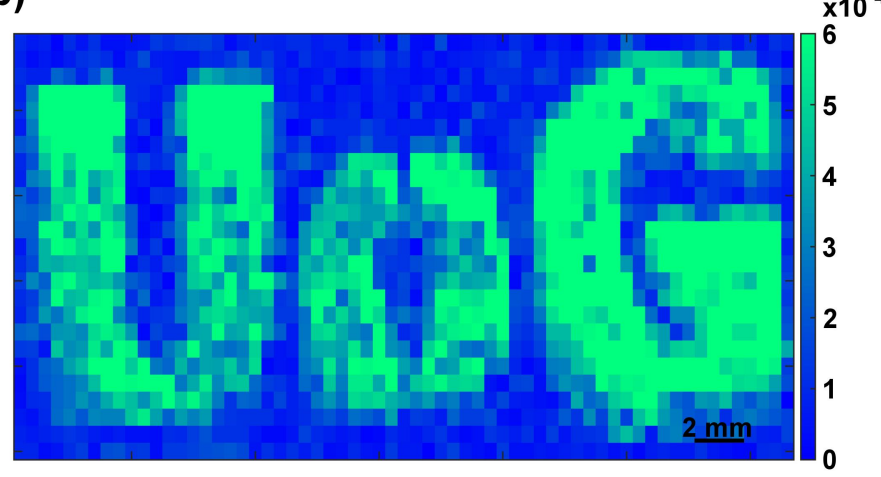

d)

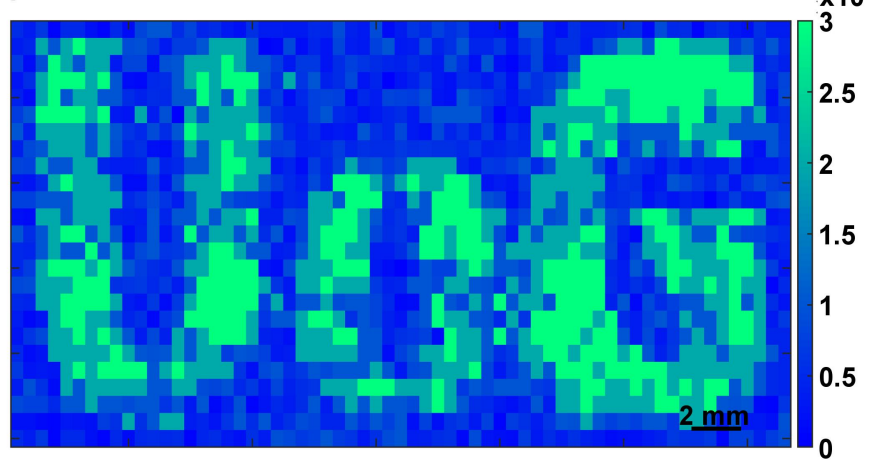

Figure 5. a) Image of aluminum cut out "UoG" object. All dimensions are in millimeters. b) THz transmission image of the "UoG" object. c) Hidden "UoG" object in a manila envelope. d) THz transmission image of the hidden object. Both images were taken by raster scanning the object and using the VOx detector operating at $2.5 \mathrm{THz}$.

\section{TOWARDS A 64 X 64 FPA}

The concept of creating high-resolution FPAs was investigated by scaling the VOx detector into a 64 x 64 FPA. Although the aforementioned CMOS chips already existed in an array format they were not thermally isolated therefore only single pixel detection was possible. A new pixel layout, shown in Figure 6a, was designed to devise a strategy to thermally isolate the pixels from each other to avoid thermal cross-talk. The broadband absorbers had two ERRs instead of three and the pixel size was increased to $40 \mu \mathrm{m} \times 40 \mu \mathrm{m}$. These changes were done to create a $26 \mu \mathrm{m} \times 26 \mu \mathrm{m}$ ground patch in M4 and a sacrificial etch layer in M3. The goal to obtain thermally isolated pixel membranes was achieved by etching the surrounding material, silicon dioxide, of the pixel and then using a wet etch to remove the sacrificial Al layer (M3) thereby forming a free-standing pixel comprised of the metamaterial absorber and the VOx microbolometer. The 
pixel membranes were supported with the stack of M1 to M6 vias in opposite corners. Figure $6 \mathrm{~b}$ shows the absorption distribution at $2.5 \mathrm{THz}$ within the pixel stack. It is clear to see that the vast majority of the incident $\mathrm{THz}$ radiation is absorbed in the inter-metal dielectric layers i.e. between M6 and M5 and M5 and M4, which are less than $3 \mu \mathrm{m}$ away from the VOx microbolometer positioned on the top surface of the chip.

Figure $6 \mathrm{c}$ shows a comparison between the simulated and experimentally measured absorption characteristics of the new pixel layout. The blue line shows the simulated spectrum for the pixel as received from the foundry. It contains the M5 and M6 ERRs, the M4 ground plane and the M3 sacrificial layer and shows $100 \%$ absorption at $2.65 \mathrm{THz}$ and $94 \%$ at $2.82 \mathrm{THz}$. The yellow line shows the simulated absorption spectrum when the sacrificial M3 Al layer and the inter-metal dielectric oxide at the perimeter of the pixel is removed and has an absorption peak of $49 \%$ at $2.67 \mathrm{THz}$. After the addition of the VOx microbolometer and protective ICP SiN layer the absorption peak red shifts to $2.58 \mathrm{THz}$ and has a magnitude of $42 \%$ (green line). The experimental results show a broadband absorption peak centered at $2.5 \mathrm{THz}$ with an absorption magnitude of $62 \%$, in reasonable agreement with the simulated results. Figure $6 \mathrm{~d}$ shows an SEM of the fully post-processed chip with the THz MM absorber and VOx bolometers thermally isolated from the underlying CMOS readout electronics.

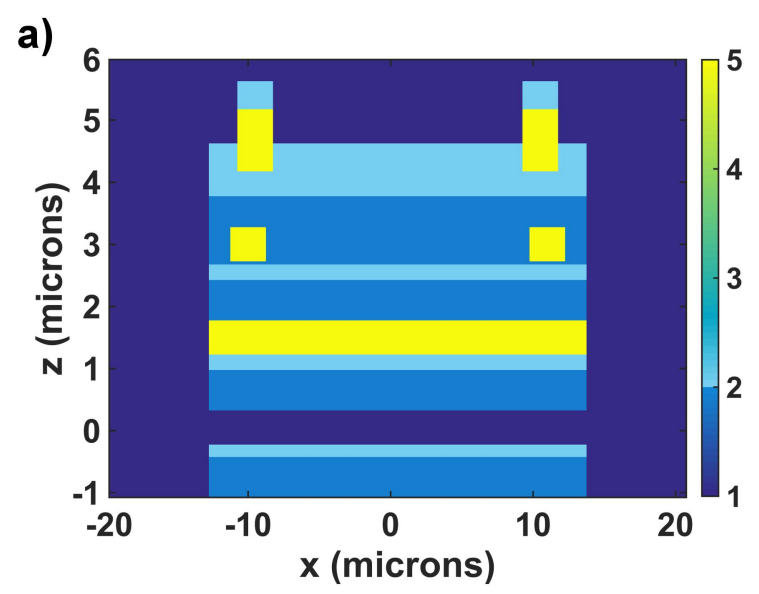

c)

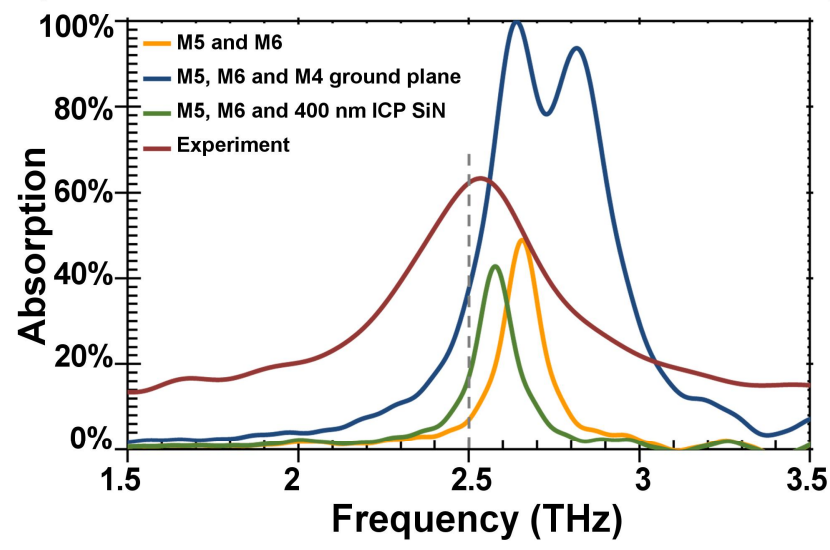

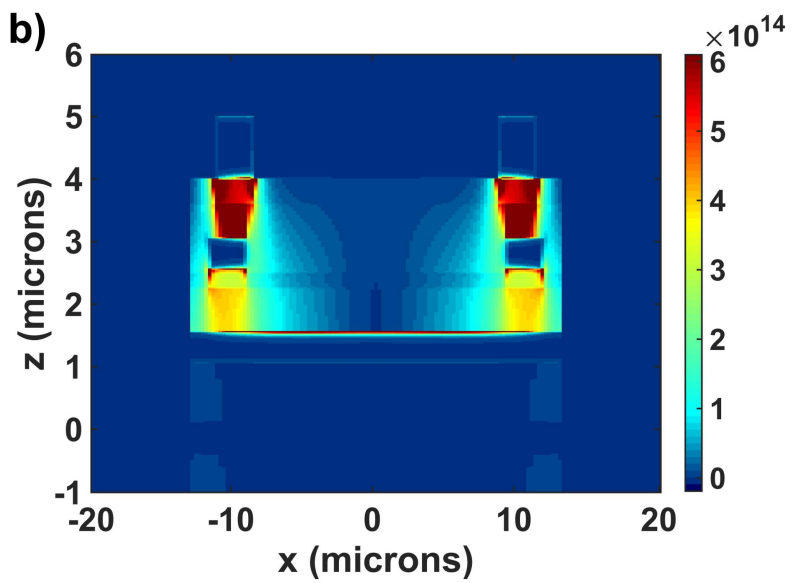

d)

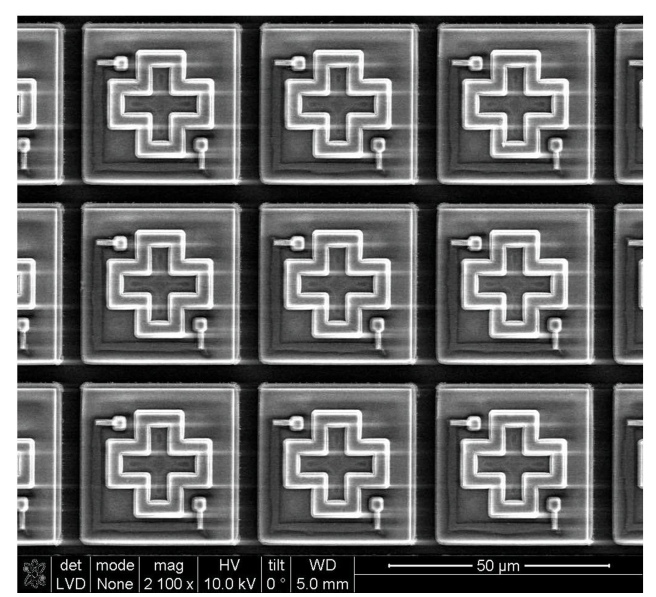

Figure 6. a) Refractive index cross-section of the new pixel layout and b) simulated absorption distribution plot. c) Spectral response of the new pixel layout showing the simulated and experimentally measured absorption response d) SEM image of the post-processed chip with patterned L shape VOx microbolometer and individual standing membranes.

\section{CONCLUSION}

The development of THz imaging technologies has been hindered due to the lack of high sensitivity and low-cost sources and detectors. Therefore, it is necessary to develop uncooled and cost-effective detectors compatible with CMOS technology to bring terahertz imaging on par with their EM neighbors. The feasibility to create MM based THz detectors monolithically integrated in the metallic and insulating layers of a commercial CMOS was presented as a low-cost 
solution for $\mathrm{THz}$ detectors. The broadband MM absorbers and microbolometer sensors, VOx and silicon pn diode, were integrated in a standard $180 \mathrm{~nm}$ six metal layer process. The performance between both $\mathrm{THz}$ detectors was compared with the VOx detector showing a higher performance. This detector was used in a single pixel transmission mode experiment demonstrating the characteristics of $\mathrm{THz}$ waves to detect concealed objects in a non-destructive manner. The detector was operated at $2.5 \mathrm{THz}$ but the flexibility of MM absorbers allows for linear scalability to other wavelengths as well as the creation of high-resolution FPAs. The combination of high-resolution FPAs with modifications of the MM absorber's shape could result in coaxial imaging for different wavelengths ${ }^{13-17}$.

\section{ACKNOWLEDGMENTS}

The authors would like to thank the staff of the James Watt Nanofabrication Centre (JWNC) at the University of Glasgow for their contributions while post-processing the chips and Texas Instruments for provision of the CMOS chips. The DOI containing the dataset associated with this research is available at Ref. 18. This work is supported by the Engineering and Physical Sciences Research Council under Grants EP/I017461/1 and EP/J018678/1.

\section{REFERENCES}

[1] FLIR Systems., "Thermal imaging A technology ready to further conquer the world," $<\mathrm{http} / / / \mathrm{www}$. flir.co.uk/cs/display/?id=51839>.

[2] Simoens, F., Meilhan, J. and Nicolas, J. A., "Terahertz Real-Time Imaging Uncooled Arrays Based on AntennaCoupled Bolometers or FET Developed at CEA-Leti," J. Infrared, Millimeter, Terahertz Waves 36(10) (2015).

[3] Dufour, D., Marchese, L., Terroux, M., Oulachgar, H., Généreux, F., Doucet, M., Mercier, L., Tremblay, B., Alain, C., Beaupré, P., Blanchard, N., Bolduc, M., Chevalier, C., D’Amato, D., Desroches, Y., Duchesne, F., Gagnon, L., Ilias, S., Jerominek, H., et al., "Review of terahertz technology development at INO," J. Infrared, Millimeter, Terahertz Waves 36(10), 922-946 (2015).

[4] Dhillon, S. S., Vitiello, M. S., Linfield, E. H., Davies, A. G., Hoffmann, M. C., Booske, J., Paoloni, C., Gensch, M., Weightman, P., Williams, G. P., Castro-Camus, E., Cumming, D. R. S., Simoens, F., Escorcia-Carranza, I., Grant, J., Lucyszyn, S., Kuwata-Gonokami, M., Konishi, K., Koch, M., et al., "The 2017 terahertz science and technology roadmap," J. Phys. D. Appl. Phys. 50(4), 43001 (2017).

[5] Ma, Y., Chen, Q., Grant, J., Saha, S. C., Khalid, A. and Cumming, D. R. S., "A terahertz polarization insensitive dual band metamaterial absorber," Opt. Lett. 36(6), 945-947 (2011).

[6] Grant, J., Ma, Y., Saha, S., Lok, L. B., Khalid, A. and Cumming, D. R. S., "Polarization insensitive terahertz metamaterial absorber," Opt. Lett. 36(8), 1524-1526 (2011).

[7] Grant, J., Ma, Y., Saha, S., Khalid, A. and Cumming, D. R. S., "Polarization insensitive, broadband terahertz metamaterial absorber," Opt. Lett. 36(17), 3476-3478 (2011).

[8] Grant, J., Escorcia-Carranza, I., Li, C., McCrindle, I. J. H., Gough, J. and Cumming, D. R. S., "A monolithic resonant terahertz sensor element comprising a metamaterial absorber and micro-bolometer," Laser Photon. Rev. 7(6), 1043-1048 (2013).

[9] Carranza, I. E., Grant, J. P., Gough, J. and Cumming, D., "Terahertz metamaterial absorbers implemented in CMOS technology for imaging applications: Scaling to large format focal plane arrays," IEEE J. Sel. Top. Quantum Electron. (2017).

[10]Escorcia, I., Grant, J., Gough, J. and Cumming, D. R. S., "CMOS terahertz metamaterial based 64 x 64 bolometric detector arrays," 2017 42nd Int. Conf. Infrared, Millimeter, Terahertz Waves, 1-2 (2017).

[11]Carranza, I. E., Grant, J., Gough, J. and Cumming, D. R. S., "Metamaterial-Based Terahertz Imaging," Terahertz Sci. Technol. IEEE Trans. 5(6), 892-901 (2015).

[12]Escorcia, I., Grant, J., Gough, J. and Cumming, D. R. S., "Uncooled CMOS terahertz imager using a metamaterial absorber and pn diode," Opt. Lett. 41(14), 3261-3264 (2016).

[13]Grant, J. P., McCrindle, I. J. H. and Cumming, D. R. S., "Multi-spectral materials: Hybridisation of optical plasmonic filters, a mid infrared metamaterial absorber and a terahertz metamaterial absorber," Opt. Express 24(4), 3451-3463 (2016).

[14]Grant, J., McCrindle, I. J. H., Li, C. and Cumming, D. R. S., "Multispectral metamaterial absorber," Opt. Lett. $39(5), 1227-1230$ (2014). 
[15]McCrindle, I. J. H., Grant, J., Drysdale, T. D. and Cumming, D. R., "Multi-spectral materials: Hybridisation of optical plasmonic filters and a terahertz metamaterial absorber," Adv. Opt. Mater. 2(2), 149-153 (2014).

[16]McCrindle, I. J. H., Grant, J. P., Gouveia, L. C. P. and Cumming, D. R. S., "Infrared plasmonic filters integrated with an optical and terahertz multi-spectral material," Phys. Status Solidi Appl. Mater. Sci. 212(8), 1625-1633 (2015).

[17]McCrindle, I. J. H., Grant, J. P., Drysdale, T. D. and Cumming, D. R. S., "Hybridization of optical plasmonics with terahertz metamaterials to create multi-spectral filters," Opt. Express 21(16), 19142-19152 (2013).

[18]"Dataset for Terahertz imagers based on metamaterial structures monolithically integrated in standard CMOS technologies.", <http://dx.doi.org/10.5525/gla.researchdata.587>. 